\title{
Synthesis and Structure Determination of Cryptomanhydride, an Uncommon Natural Terpenic Anhydride
}

\author{
Wen-Chiung Su, Jim-Min Fang, and Yu-Shia Cheng* \\ Department of Chemistry, National Taiwan University, Taipei, Taiwan 106, Republic of China
}

\begin{abstract}
A novel terpenic anhydride, namely cryptomanhydride, was isolated from the leaves of Cryptomeria japonica; its absolute configuration was determined by a partial synthesis from hinokiol.
\end{abstract}

The Japanese cedar, Cryptomeria japonica D. Don., is a widely distributed conifer called 'sugi' in Japanese. ' From the ethyl acetate-soluble part of the acetone extract of the leaves, we isolated a novel terpenic anhydride 1 , namely cryptomanhydride. ${ }^{2}$ This compound has a unique skeleton incorporating an abietane diterpene and a $p$-cymene monoterpene. This is the first report of such uncommon terpenic anhydride in nature. ${ }^{3}$

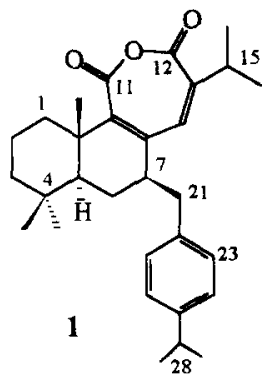

$$
\begin{aligned}
& \text { Oil, }[\alpha]_{\mathrm{D}}{ }^{30}+370^{\circ}(\mathrm{MeOH} ; c 0.4) \\
& \operatorname{TLC}\left(\text { Merck silica gel } 60 \mathrm{~F}, \mathrm{CH}_{2} \mathrm{Cl}_{2} / \text { hexane }=1: 2\right) R_{f} 0.37 \\
& \begin{aligned}
& \mathrm{UV}(\mathrm{MeOH}): 255 \mathrm{~nm}(\varepsilon 8500), 236 \mathrm{~nm}(\varepsilon 7000), \\
& 219 \mathrm{~nm}(\varepsilon 15200), 217 \mathrm{~nm}(\varepsilon 15000)
\end{aligned} \\
& \begin{aligned}
\mathrm{CD}(\mathrm{MeOH}):: & {[\theta]_{266}+61900,[\theta]_{236}+38300, } \\
& {[\theta]_{223}+32300,[\theta]_{203}+22000 }
\end{aligned} \\
& \text { HRMS for } \mathrm{C}_{30} \mathrm{H}_{40} \mathrm{O}_{3} \text { requires } 448.2979 ; \text { found } 448.2977
\end{aligned}
$$

The structure of 1 was determined by analysis of its spectral properties. The parent peak appearing at $\mathrm{m} / \mathrm{z}$ 448.298 indicated the molecular formula $\mathrm{C}_{30} \mathrm{H}_{40} \mathrm{O}_{3}$. The anhydride moiety was inferred from the IR absorptions at 1775 and $1733 \mathrm{~cm}^{-1}$ as well as from the ${ }^{13} \mathrm{C}$ signals at $\delta 161.6(\mathrm{C}-11)$ and $162.0(\mathrm{C}-12)$. The ${ }^{1} \mathrm{H}$ and ${ }^{13} \mathrm{C}$ resonances (Table 1) were assigned by assistance of the $\mathrm{C}-\mathrm{H}$ COSY and HMBC spectra. Four aromatic protons on the para-disubstituted phenyl ring occurred at $\delta 7.04(d, J=8 \mathrm{~Hz})$ and $7.15(d, J=8 \mathrm{~Hz})$. The olefinic proton (H-14) occurring at a low field $\delta 6.16(s)$ was attributable to the conjugated system. The cymenyl group is on equatorial position as the axial H-7 at $\delta 2.60$ exhibited a large coupling constant $J_{6 \beta, 7}=$ $13 \mathrm{~Hz}$.

Compound 1 exhibited a positive Cotton effect in the CD spectrum. Its absolute configuration was determined by a partial synthesis from hinokiol (3), a constituent of known configuration isolated from the same plant $C$. japonica. The phenol moiety of hinokiol was selectively methylated (Scheme I). The product 4 
Table $1 .{ }^{1} \mathrm{H}$ and ${ }^{13} \mathrm{C}$ NMR spectral data of $1\left(\mathrm{CDCl}_{3} \text { solution, } \delta \text { value in ppm, } J \text { values in } \mathrm{Hz}\right)^{*}$

\begin{tabular}{rrllrrl}
\hline & \multicolumn{1}{c}{$\mathrm{C}$} & $\mathrm{H}$ & & $\mathrm{C}$ & $\mathrm{H}$ \\
\hline 1 & 36.4 & $1.23(d d d, J 3.5,12,12.5)$ & 14 & 133.1 & $6.16(s)$ \\
& & $1.83(d d d, J 3,5,12.5)$ & 15 & 32.2 & $2.82(\operatorname{sept}, J 7)$ \\
2 & 18.6 & $1.50(m), 1.64(m)$ & 16 & 20.6 & $1.07(d, J 7)$ \\
3 & 41.5 & $1.20(m)$ & 17 & 22.1 & $1.09(d, J 7)$ \\
& & $1.46(d d d, J 3,4.5,12.5)$ & 18 & 33.4 & $0.89(s)$ \\
4 & 33.2 & & 19 & 21.4 & $0.81(s)$ \\
5 & 46.1 & $1.19(d d, J 4,12)$ & 20 & 20.2 & $1.33(s)$ \\
6 & 21.7 & $1.58(d d d, J 2.5,4,12)$ & 21 & 40.3 & $2.44(d d, J 9,13)$ \\
& & $1.60(d d d, J 12,12,13)$ & & & $2.67(d d, J 3.5,13)$ \\
7 & 41.5 & $2.60(d d d d, J 2.5,3.5,9,13)$ & 22 & 136.9 & \\
8 & 138.2 & & 23,27 & 129.1 & $7.04(d, J 8)$ \\
9 & 142.9 & & 24,26 & 126.6 & $7.15(d, J 8)$ \\
10 & 39.3 & & 25 & 147.1 & \\
11 & 161.6 & & 28 & 33.7 & $2.83(s e p t, J 7)$ \\
12 & 162.0 & & 29,30 & 24.0 & $1.22(d, J 7)$ \\
13 & 141.9 & & & & \\
\hline
\end{tabular}

* The assignments of ${ }^{1} \mathrm{H}(300 \mathrm{MHz})$ and ${ }^{13} \mathrm{C}(75 \mathrm{MHz})$ signals were confirmed by the $\mathrm{H}-\mathrm{H}$ COSY, C-H COSY, HMBC and $J$-resolved spectra as well as by the nOe experiments.

was treated with $\mathrm{PBr}_{3}$ to give the corresponding bromides 5 (two 3-epimers), which were subsequently reduced with $\mathrm{Bu}_{3} \mathrm{SnH}_{/} / \mathrm{Et}_{3} \mathrm{~B}$ to afford ferruginol methyl ether $(6)$. The $(5 S, 10 S)$-configuration of ferruginol methyl ether has been determined. ${ }^{4}$ The optical rotation of $6,[\alpha]^{20}{ }_{D}+37.5^{\circ}\left(\mathrm{CHCl}_{3} ; c 3.3\right)$, was in agreement with the reported value, $[\alpha]_{\mathrm{D}}+35.9^{\circ}\left(\mathrm{CHCl}_{3}\right)$.

Direct introduction of cymenyl group at $\mathrm{C}-7$ of compound 6 by means of metalation-alkylation may be complicated by side-products derived from ortho-metalations. Wittig reaction of ketone 7 with the phosphorus ylide, prepared from 7-bromocymene, $\mathrm{Ph}_{3} \mathrm{P}$ and $\mathrm{BuLi}$, failed. Cymenyl substituent was successfully introduced by a three-step sequence: (i) oxidation of 6 with pyridinium chlorochromate, giving ketone 7 (sugiol methyl ether, $95 \%$ ) along with a small amount of enone 8; (ii) addition of the Grignard reagent prepared from 7bromocymene onto ketone 7 , giving 9 as a single product $(90 \%)^{5}$; and (iii) catalytic hydrogenation of 9 in the presence of hydrochloric acid, giving 11 (two 7-epimers). Alternatively, 9 was dehydrated to give alkene 10, which underwent hydrogenation to yield 11. By analysis of the ${ }^{1} \mathrm{H}$ NMR spectrum, compound 11 consisted of two 7-epimers (3:2) inseparable by HPLC.

According to the reported method, ${ }^{6}$ the isomeric mixture of 11 was treated with $m$-chloroperbenzoic acid ( 3 equiv) in darkness for 5 days to give cryptomanhydride $1(15 \%)$ having $(5 S, 7 R, 10 S)$-configuration and the $7 S$-epimer $2^{7}(10 \%)$ along with recovery of $11(30 \%)$.

Acknowledgment: We are grateful to the National Science Council for financial support (Grant NSC83-0208M002-95) 


\section{Scheme I}

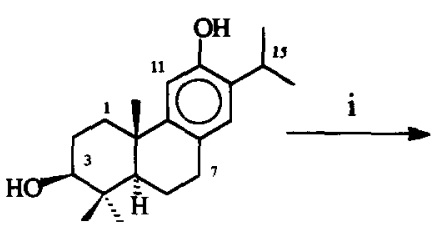

3<smiles>COc1cc2c(cc1C(C)C)CC[C@H]1[C@@H](C)[C@H](O)CC[C@]21C</smiles>

4<smiles>COc1cc2c(cc1C(C)C)CC[C@@H]1[C@@H](C)[C@H](Br)CC[C@@]21C</smiles>

5<smiles>CC#CCCCCC#N</smiles>

6<smiles>COc1cc2c(cc1C(C)C)C(=O)C[C@H]1[C@@H](C)CCC[C@]21C</smiles>

7<smiles>COc1cc2c(cc1C(C)C)C(=O)C=C1C(C)(C)CCC[C@]12C</smiles>

8<smiles>COc1cc2c(cc1C(C)C)C(Cc1ccc(C(C)C)cc1)=CC1C(C)(C)CCCC21C</smiles>

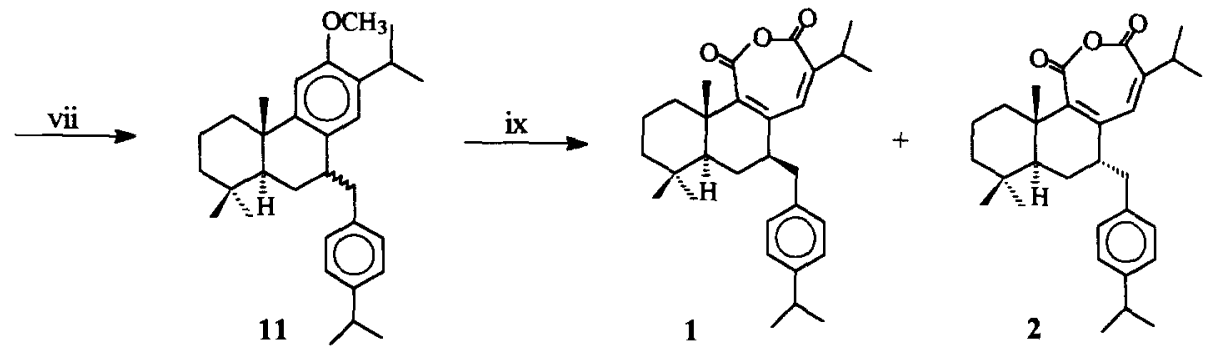

Reagents and conditions: (i) $\mathrm{CH}_{3} \mathrm{I}, \mathrm{Me}_{2} \mathrm{CO}, \mathrm{K}_{2} \mathrm{CO}_{3}, \mathrm{H}_{2} \mathrm{O}$, reflux, $16 \mathrm{~h}$; $95 \%$. (ii) $\mathrm{PBr}_{3}, \mathrm{Et}_{2} \mathrm{O}, 20{ }^{\circ} \mathrm{C}, 16 \mathrm{~h}$; then $5 \% \mathrm{NaHCO}_{3} ; 96 \%$. (iii) $\mathrm{Et}_{3} \mathrm{~B}$, benzene, $\mathrm{Bu}_{3} \mathrm{SnH}, 20^{\circ} \mathrm{C}, 6 \mathrm{~h}$; then $\mathrm{Et}_{3} \mathrm{~N} ; 96 \%$. (iv) $\mathrm{PCC}$, benzene, reflux, $16 \mathrm{~h}$; 95\%. (v) $\mathrm{Mg}$, THF, $0{ }^{\circ} \mathrm{C}$ to $20{ }^{\circ} \mathrm{C}, 2 \mathrm{~h}$; then $\mathrm{NH}_{4} \mathrm{Cl}, \mathrm{H}_{2} \mathrm{O} ; 90 \%$. (vi) $p$ - $\mathrm{CH}_{3} \mathrm{C}_{6} \mathrm{H}_{4} \mathrm{SO}_{3} \mathrm{H}$, benzene, reflux, $16 \mathrm{~h}$; $100 \%$. (vii) $10 \% \mathrm{Pd} / \mathrm{C}, \mathrm{MeOH}, \mathrm{H}_{2}, 20{ }^{\circ} \mathrm{C}, 16 \mathrm{~h} ; 100 \%$. (viii) $10 \% \mathrm{Pd} / \mathrm{C}, \mathrm{MeOH}, \mathrm{H}_{2}, 10 \% \mathrm{HCl}, 20^{\circ} \mathrm{C}, 16 \mathrm{~h}$; 95\%. (ix) MCPBA, $\mathrm{CH}_{2} \mathrm{Cl}_{2}, 20{ }^{\circ} \mathrm{C}, 5$ days; 1 (15\%), 2 (10\%). 


\section{REFERENCES AND NOTES}

1. Gan, W. S. (1958) Manual of Medicinal Plants in Taiwan. Nat. Res. Inst. Chin. Med.: Taipei, 1958, Vol. 1, 54-55. A voucher specimen is deposited in our laboratory. We previously isolated a series of sesquiterpenes, diterpenes (abietane-, kaurane- and labdane-types) and triterpenes from this plant, see Su, W.-C.; Fang, J.-M.; Cheng, Y.-S. Phytochemistry 1993, 34, 779; ibid. 1994, 35, 1279; ibid. 1994, 37, 1109.

2. The system name of 1 is $(6 R, 7 \mathrm{a} S, 11 \mathrm{a} S)$-4-isopropyl-6-(4-isopropylphenyl)methyl-6,7,7a,8,9,10,11,11aoctahydro-8,8,1 1a-trimethylnaphth[1,2-c]oxepin-1,3-dione. We use the numbering of abietanes for 1 in this manuscript.

3. Three norabietane anhydrides have been found in the Chinese drug Danshen, Salvia multiorrhiza. see Chang, H. M.; Choang, T. F.; Chui, K. Y.; Hon, P. M.; Lee, C. M.; Mak, T. C. W.; Wong, H. N. C. J. Chem. Res. (S) $1990,114$.

4. Matsumoto, T.; Usui, S. Bull. Chem. Soc. Jpn. 1979, 52, 212.

5. Compound $9\left(\mathrm{C}_{31} \mathrm{H}_{44} \mathrm{O}_{2}\right),[\alpha]^{20}{ }_{\mathrm{D}}-31.5^{\circ}\left(\mathrm{CHCl}_{3} ; c\right.$ 3.3), was homogenous in the ${ }^{1} \mathrm{H}$ and ${ }^{13} \mathrm{C} \mathrm{NMR}$ spectra. The 7-hydroxyl group of 9 was considered to be on the axial position as evidenced by (i) irradiation of $\mathrm{H}-21$ causing a 7\% NOE of $\mathrm{H}-14$; (ii) an intense fragment $\left[\mathrm{M}-\mathrm{H}_{2} \mathrm{O}\right]^{+}$at $\mathrm{m} / \mathrm{z} 430$ occurring in the mass spectrum of 9 without appearance of the parent peak.

6. Bendall, J. G.; Cambie, R. C.; Grimsdale, A. G.; Rutledge, P. S.; Woodgate, P. D. Aust. J. Chem. 1992, $45,1063$.

7. Compound 2: Oil, $[\alpha]^{15}{ }_{\mathrm{D}}+110^{\circ}\left(\mathrm{CDCl}_{3} ; c 0.3\right)$. TLC $\left(\mathrm{CH}_{2} \mathrm{Cl}_{2} /\right.$ hexane $\left.=1: 2\right) R_{f} 0.32$. IR $v^{\text {neat }}{ }_{\max }$ $\mathrm{cm}^{-1}: 1775,1734,1507,1455$. UV $\lambda^{{ }^{\mathrm{MeOH}}{ }_{\max } \mathrm{nm}(\varepsilon): 252(8400), 236(7600), 219(15400) .}{ }^{1} \mathrm{H}$ NMR $\left(\mathrm{CDCl}_{3}, 300 \mathrm{MHz}\right): \delta 0.80(s, \mathrm{H}-18), 0.82(s, \mathrm{H}-19), 1.00(d, J=7 \mathrm{~Hz}, \mathrm{H}-16), 1.02(d, J=7 \mathrm{~Hz}, \mathrm{H}-$ 17), $1.11(s, \mathrm{H}-20), 1.22(d, J=7 \mathrm{~Hz}, \mathrm{H}-29,30), 2.64(m, \mathrm{H}-7), 2.72(d d, J=7,13.5 \mathrm{~Hz}, \mathrm{H}-21), 2.82$ (sept, $J=7 \mathrm{~Hz}, \mathrm{H}-28), 2.87$ (sept, $J=7 \mathrm{~Hz}, \mathrm{H}-15), 2.90$ (dd, $J=6,13.5 \mathrm{~Hz}, \mathrm{H}-21), 6.34$ (s, H-14), $7.06(d, J=8 \mathrm{~Hz}, \mathrm{H}-23,27), 7.15(d, J=8 \mathrm{~Hz}, \mathrm{H}-24,26) .{ }^{13} \mathrm{C} \mathrm{NMR}\left(\mathrm{CDCl}_{3}, 75 \mathrm{MHz}\right): \delta 18.5(\mathrm{C}-2)$, 21.0 (C-20), 21.2 (C-16, 17), 21.6 (C-19), 24.0 (C-29, 30), 25.6 (C-6), 32.4 (C-15), 32.8 (C-18), 33.3 (C-4), 33.7 (C-28), 36.9 (C-1), 38.2 (C-10), 40.4 (C-7), 40.7 (C-21), 41.5 (C-3), 49.7 (C-5), 126.7 (C24, 26), 128.9 (C-23, 27), 130.7 (C-14), 136.3 (C-8), 136.8 (C-22), 141.7 (C-13), 143.4 (C-9), 147.2 (C-25), 161.4 (C-11), 162.3 (C-12). EIMS (70eV) m/z (rel. int.) 448 [M] (5), 315 (25), 271 (10), 243 (4), 133 (100), 117 (18), 105 (17). $\mathrm{HRMS}$ for $\mathrm{C}_{30} \mathrm{H}_{40} \mathrm{O}_{3}$ requires 448.2979; found 448.2975. 